\title{
Rheology of dense suspensions of non colloidal particles
}

\author{
Élisabeth Guazzelli ${ }^{1, \star}$ \\ ${ }^{1}$ Aix Marseille Univ, CNRS, IUSTI, Marseille, France
}

\begin{abstract}
Dense suspensions are materials with broad applications both in industrial processes (e.g. waste disposal, concrete, drilling muds, metalworking chip transport, and food processing) and in natural phenomena (e.g. flows of slurries, debris, and lava). Despite its long research history and its practical relevance, the mechanics of dense suspensions remain poorly understood. The major difficulty is that the grains interact both by hydrodynamic interactions through the liquid and by mechanical contact. These systems thus belong to an intermediate regime between pure suspensions and granular flows. We show that we can unify suspension and granular rheology under a common framework by transferring the frictional approach of dry granular media to wet suspensions of spherical particles. We also discuss non-Newtonian behavior such as normal-stress differences and shear-induced migration. Beyond the classical problem of dense suspension of hard spheres which is far from being completely resolved, there are also entirely novel avenues of study concerning more complex mixtures of particles and fluids such as those involving other types of particles (e.g. fibers) or non-Newtonian fluids that we will also address.
\end{abstract}

\section{Introduction}

Dense suspensions are materials with broad applications both in industrial processes (e.g. waste disposal, concrete, drilling muds, metalworking chip transport, and food processing) and in natural phenomena (e.g. flows of slurries, debris, and lava). Despite its long research history and its practical relevance, the mechanics of dense suspensions remain poorly understood.

In order to understand their flowing behavior, it is desirable to know their response to imposed forces and motions at their boundary. The fundamental problem is then to determine the rheological properties of these media (considered as equivalent homogeneous materials) from a knowledge of the mechanics of the particles and the interstitial fluid. In other words, the key problem is to understand the relationship between the macroscopic or bulk properties of the medium and its microscopic structure at these large concentrations.

The major difficulty of dense particulate flows is that the grains interact both by hydrodynamic interactions through the liquid and by mechanical contact. Dense or highly concentrated particulate flows indeed belong to an intermediate regime between pure suspensions and granular flows. The complex nature of these particle interactions greatly contribute to the lack of understanding of these systems.

The present contribution aims at providing a review of recent work on the rheology of dense suspensions of non colloidal particles. These studies were done in collaboration with F. Boyer, J. E. Butler, E. Couturier, S.

\footnotetext{
^e-mail: Elisabeth.Guazzelli@univ-amu.fr
}

Dagois-Bohy, L. M. Davidson, S. Hormozi, O. Pouliquen, P. R. Nott, S. Shaikh, B. Snook, S. Strednak, F. Tapia.

\section{Suspension viscosity}

Adding suspended particles to a fluid increases its viscosity. This is known for a Newtonian fluid since the seminal work of Einstein demonstrating that the viscosity of the mixture is increased above that of the suspending fluid [1]. The suspension viscosity increases with increasing particle concentration and diverges at the jamming transition where the particle concentration reaches a maximum value, see e.g. [2-5].

To be more precise, for a suspension of neutrallybuoyant solid spheres under steady shearing conditions, the linearity of the Stokes equations leads to a viscous scaling of the shear stress, $\tau=\eta_{s}(\phi) \eta_{f} \dot{\gamma}$, where $\dot{\gamma}=\sqrt{2 \mathbf{E}: \mathbf{E}}$ is the shear rate (where $\mathbf{E}$ is the rate of strain) and $\eta_{f}$ is the viscosity of the suspending Newtonian fluid. For monodisperse suspensions of non-Brownian rigid spheres, the dimensionless effective shear viscosity, $\eta_{s}$, only depends on the particle volume fraction, $\phi$, and increases with increasing $\phi$, diverging at maximum packing fraction, $\phi_{m}$.

\section{Non-Newtonian behavior: Normal stresses}

This quasi-Newtonian behavior of the shear stress does not fully describe the rheology of suspensions, as it does not account for the possible existence of normal stress differences. These normal stress differences also scale viscously 
in Stokes flows and, since the normal stresses do not depend on the sign of the shear rate, are proportional to the modulus of the shear stress, $|\tau|$. These normal stress differences can be written as $N_{1}=\alpha_{1}|\tau|$ and $N_{2}=\alpha_{2}|\tau|$, where $N_{1}$ and $N_{2}$ are the first and second normal stress differences. The ratios of normal-stress differences to shear stress, $\alpha_{1}$ and $\alpha_{2}$, are called the normal stress difference coefficients. They are sole functions of $\phi$ and do not diverge at $\phi_{m}$ since the normal stress differences and the shear stress present the same divergence when approaching jamming at $\phi_{m}[6]$. .

Normal stresses are difficult to measure using standard rheological tools. They were chosen to be inferred from measurements of the deflection of the free-surface in a Weissenberg, or rotating-rod, geometry and in a tiltedtrough $[7,8]$. The first method is well-known in polymers as the Weissenberg or rod-climbing effect, see figure $1(a)$. For suspensions of spheres, the climbing is down instead of up the rod and measurement of the free-surface profile provides a combination of the normal stress differences. The second tilted-trough method provides the second normal stress difference in isolation, see figure $1(b)$. Combining both methods yields a complete measurement of the two differences which are both linear in the modulus of the shear rate. These approaches have some significant advantages over using a standard rheometer as confinement effect can be reduced and sensitivity improved [7-9].

For suspensions of spheres, the second normal stress difference, $N_{2}$, is observed to be negative and to increase with increasing particle volume fraction (growing especially quickly for $\phi \gtrsim 0.20$ ), see e.g. [5]. The properties of the first normal stress difference are more elusive. The magnitude of $N_{1}$ is unquestionably much smaller than that of $N_{2}$, but assessing the sign is difficult. Using these non conventional rheological tools (rotating-rod and tiltedtrough), the value is found too close to zero to determine whether it is negative, positive or null within experimental accuracy. Some experiments using more conventional rheometry find that $N_{1}$ is quite small and negative [10-13], while others report positive values [14]. Some recent studies point to the effect of polydispersity [15] and of confinement [16] which may explain these discrepancies.

Whereas the whole suspension, i.e. the mixture of the particles and the fluid, is incompressible, the particle phase is not. There exists a pressure coming from the dispersed particulate phase that could develop. It has been termed particle pressure (or more generally particle normal-stress) and is analogous to the osmotic pressure exerted by both colloidal particles and dissolved molecules, see e.g. [17, 18]. This particle normal stress, $P$, also scales viscously and is linear in the modulus of the shear rate since it must be independent of the sign of the shear rate as previously noted for the normal stresses of the whole suspension. It can be written as $P=\eta_{n}(\phi) \eta_{f} \dot{\gamma}$, where $\dot{\gamma}$ is conveniently defined in an invariant form as $\dot{\gamma}=\sqrt{2 \mathbf{E}: \mathbf{E}}$, where $\mathbf{E}$ is the rate of strain. The dimensionless effective normal viscosity, $\eta_{n}$, is again a sole function of $\phi$ and presents the same divergence as $\eta_{s}(\phi)$ when approaching jamming at $\phi_{m}[6]$. (a)

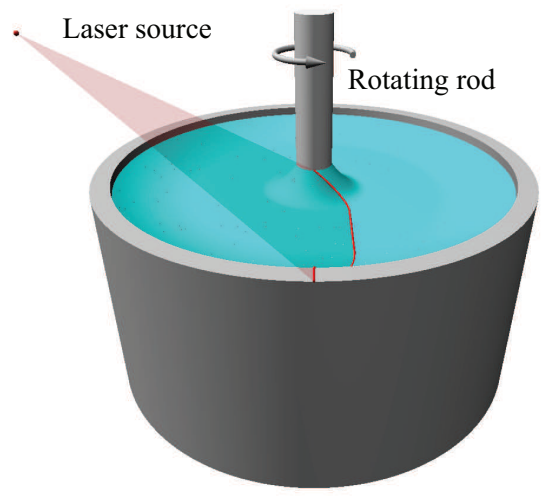

(b) Laser source

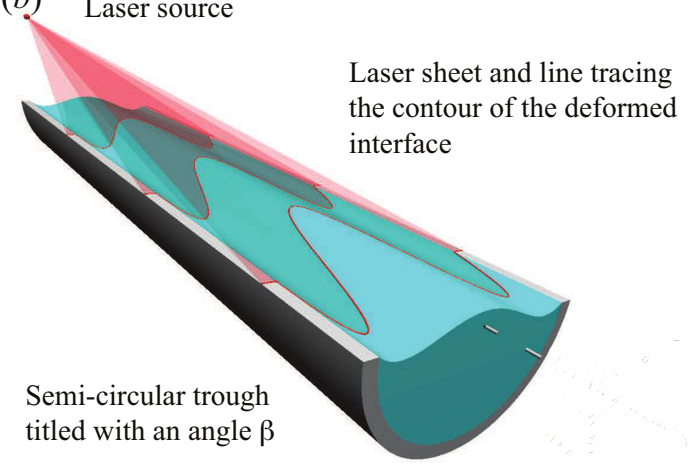

Figure 1. Sketches adapted from figure 2 of [9] of the (a) rotating-rod and (b) tilted-trough flows. Laser sheets, projected at low angles onto the surfaces of the flowing suspensions, enable quantification of the surface deformations and subsequently to the determination of the normal stress differences.

Quantitative measurements of the particle-phase stresses are not easily performed and only few experiments are reported in the literature [19-21]. The imposedpressure approach of [20] will be discussed in section 5 . The particle pressure is also of crucial importance in the understanding of shear-induced migration which is discussed in the following section 4 .

\section{Irreversible dynamics: Shear-induced migration}

Shear-induced migration is a manifestation of irreversible dynamics in shearing flows of non-Brownian suspension. This irreversibility may be unexpected as, at low Reynolds numbers, the velocity and pressure fields are supposed to be governed by the linear and reversible Stokes equations. It is due to the combined effects of hydrodynamics interactions between the particles and non-hydrodynamic forces such as close contact interactions (short-ranged repulsive forces or small roughnesses).

Shear-induced migration drives particles from regions of high to low shear rate. This phenomenon was first clearly identified in a Couette rheometer [22] and moti- 


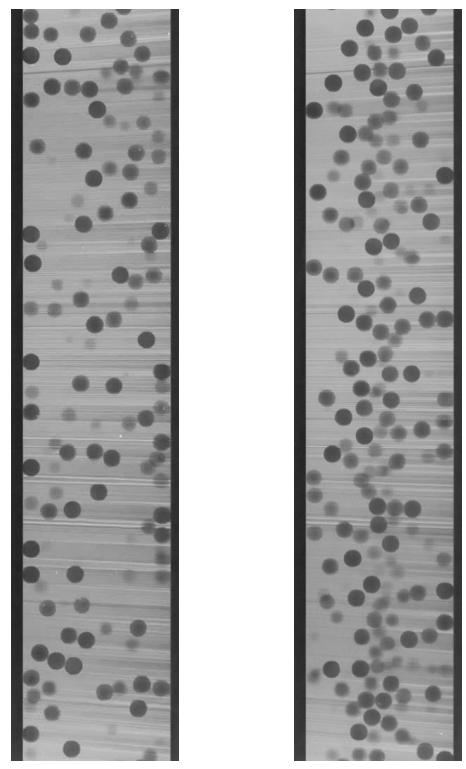

Figure 2. Images of a suspension, consisting of neutrally buoyant spheres, at a bulk volume fraction of $20 \%$ subjected to large oscillating displacements at low Reynolds number in a pipe, adapted from movie 1 of [24]. Particle volume fraction and velocity can be measured during the oscillations by matching the refractive index of the fluid to that of the particles and using fluorescence to distinguish the particle and fluid phases for imaging with a thin laser sheet. The left image corresponds to the initially mixed suspension and the right image to the suspension after 32 oscillations where fully-developed migration toward the centerline is observed.

vated a large body of research due to its implication on the characterization of suspension rheology, see e.g. [3-5]. In pressure-driven Poiseuille flow, the particles migrate toward the centerline, see figure 2. Again, since the first observation of such inhomogeneities in suspension pipe flows [23], several experimental studies have been performed to measure migration in pressure-driven flow in a pipe or a channel, see e.g. [24] and references therein.

Since the first observation of shear-induced migration, two types of migration models have been proposed. Early efforts used a diffusion model, in which the particle migration flux was expressed in terms of the gradients of the particle concentration and shear rate. This diffusion model is successful in predicting migration in wide-gap Couette and pressure-driven Poiseuille flows, but fails to predict the absence of migration in curvilinear torsional flows, see e.g. [6] and references therein. A more recent and rather successful model, termed the suspension balance model, relates the migration flux to the rheology of the suspension [25]. This modeling uses a two-phase approach of the suspension and finds that the particle migration flux is driven by the divergence of the normal stress of the particle phase [6, 25-27]. This model requires correlations for the particle phase stress which cannot be easily obtained experimentally. When using realistic correlations, the suspension balance model qualitatively supports the experimental observations but presents however some quantitative discrepancies [24].

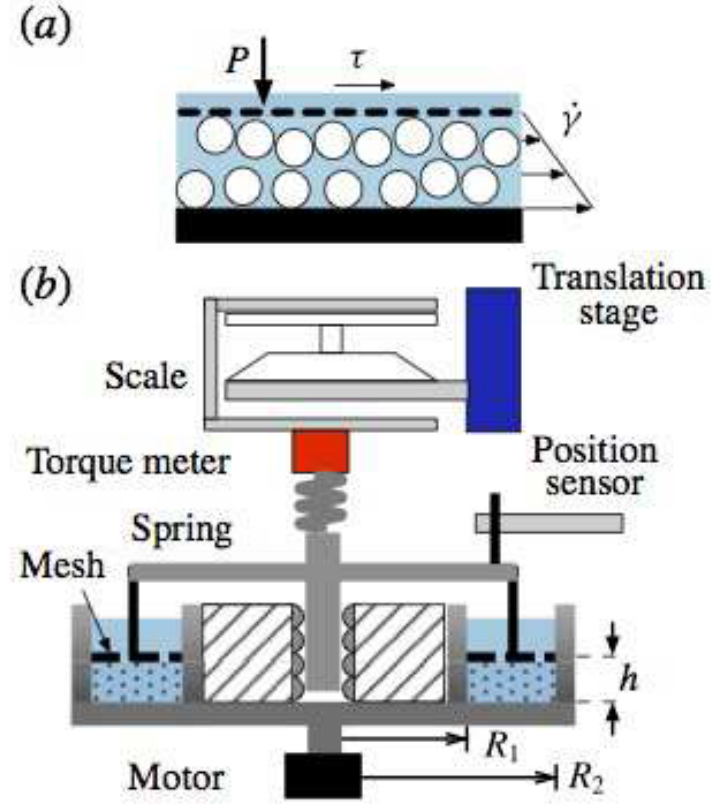

Figure 3. (a) Principle of the pressure-imposed rheometry: the porous plate exerts a pressure $(P)$ on the grains while the suspension is sheared $(\dot{\gamma})$ and the shear stress $(\tau)$ is measured. (b) Sketch of the pressure-imposed shear cell, adapted from figure 2 of [29]. The shear cell consists of (i) a bottom annular cylinder (of radii $R_{1}=43.95 \mathrm{~mm}$ and $R_{2}=90.28 \mathrm{~mm}$ ) that contains the suspension and rotates and (ii) a top cover plate that can be moved vertically and which is porous to enable fluid flow through it. From the measurements of the position of the top plate $h$, the rotation rate of the bottom annulus, and the normal force and torque exerted on the top plate, it is possible to extract $\phi, \dot{\gamma}, P$, and $\tau$.

Another avenue to study inhomogeneities in suspension flows is to perform discrete particle simulations. Earlier simulations of the pressure-driven flow in a twodimensional channel of a suspension were conducted using Stokesian Dynamics [25]. Numerical capabilities are expanding rapidly and new methods such as the force coupling method [28] are providing effective information on the migration process in agreement with experiments [24].

\section{A frictional approach: Unifying suspension and granular rheology}

As explained in sections 2 and 3, for a suspension consisting of neutrally-buoyant spheres in a Newtonian fluid of viscosity, $\eta_{f}$, the scaling of the stresses is viscous, and consequently, when the suspension is sheared under a constant shear rate, $\dot{\gamma}$, at concentration, $\phi$, the shear stress is $\tau=\eta_{s}(\phi) \eta_{f} \dot{\gamma}$ and the particle pressure is $P=\eta_{n}(\phi) \eta_{f} \dot{\gamma}$. The rheology thus reduces to the knowledge of the two functions $\eta_{s}(\phi)$ and $\eta_{n}(\phi)$ which are known to diverge in a similar way at maximum volume fraction, $\phi_{m}$.

There is an equivalent approach to this classical view in terms of effective viscosities which is coming from the rheology of dry granular materials and hinges on a frictional view of the problem, see e.g. [30]. When an assem- 
bly of particles is subjected to steady shear under a confining particle pressure, $P$, there is only one dimensionless control parameter, a dimensionless shear rate which can be interpreted as the ratio of the time scale for particles to rearrange due to the pressure, $P$, to the time scale of the flow, $\dot{\gamma}^{-1}$, see figure $3(a)$. The friction, $\mu=\tau / P$, and the volume fraction, $\phi$, are sole function of this dimensionless number. In the case of immersed granular media for which viscous forces are dominant, the dimensionless number is $J=\eta_{f} \dot{\gamma} / P$ and is viscous in contrast to dry granular flows where it is inertial. Using an original pressureimposed shear cell such as sketched in figure $3(b), \mu(J)$ and $\phi(J)$ has been found once again to collapse onto universal curves [20].

Describing the flow in terms of an effective friction coefficient thus works for both granular flows and suspensions, and therefore unifies suspension and granular rheology. This alternative way of looking at suspensions enabled to circumvent the divergence observed in volumeimposed rheometry and provided examination of the rheology extremely close to the jamming transition. In particular, this approach yields accurate measurements of the particle pressure, a quantity not often easily captured as mentioned earlier at the end of section 3 .

\section{Toward more complex particulate systems}

The pressure-imposed rheometry presented in section 5 complemented by the free-surface-profilometry measurements giving access to normal stresses differences presented in section 3 provide a full description of the stress tensor of dense particulate system. These unconventional rheological tools offer a brand new perspective of analysis which can be applied to more complex particulate systems and offer new avenues of research.

Studies have been undertaken to analyze the influence of the shape of the particles (e.g. large-aspect-ratio particles such as fibers) on the rheological behaviors. In particular, measurements of normal stress differences have been reported for suspensions of rigid, non-Brownian fibers at high concentrations [9]. The second normal stress difference is found to be negative and its magnitude increases with increasing concentration and decreasing aspect ratio. The first normal stress difference is positive and its magnitude is approximately twice that of the second normal stress difference. Numerical simulations which account for both hydrodynamic and contact contributions reveal that the contact interactions are largely responsible for the observed normal stress differences [9]. The rheological behaviors observed for suspensions of fibers, which are qualitatively different from those for suspensions of spheres, have implications for flows of fibers in more general situations (e.g. in pressure-driven flows).

Systematic investigations have been also performed to examine how the macroscopic rheology close to the jamming transition is influenced by the nature of the suspending fluid. In particular, the rheology of non colloidal suspensions composed of a yield-stress fluid and of neutrally-buoyant solid spheres has been explored in the dense regime [29]. The rheological measurements are in agreement with a model based on scaling arguments and homogenization methods $[31,32]$. A striking result is that all the data including a Newtonian test case show a perfect collapse of the friction, $\tau / P=\mu(\phi)$. This shows that the constitutive laws close to jamming has a form similar to that for a Newtonian suspending fluid and thus demonstrates unambiguously that the dynamics of the particles close to jamming are mainly controlled by geometrical constraints and are independent of the suspending fluid. This scaling approach has been further tested by inferring the lever function relating the local shear rate seen by the particles to the macroscopic shear rate imposed by the rheological flow and by showing that an excellent collapse is again obtained with a sole dependence in $\phi$. The properties of the viscoplastic suspensions can be satisfactorily modeled as that of a Herschel-Bulkley fluid with an exponent equal to that of the suspending fluid. The dimensionless effective yield-stress and viscosity (to be more precise, consistency) are found to be sole functions of $\phi$ that can be deduced from the knowledge of the lever function and the dimensionless effective viscosity, $\eta_{s}(\phi)$, of a test suspension having a Newtonian suspending fluid. This study offers new perspective in the study of even more complex particulate systems as the knowledge of the rheological constitutive laws in the Newtonian case and the calculation of the lever function relating the local to the macroscopic shear rate seem to be the sole ingredients needed to infer the rheological properties of these suspensions in the dense regime. This may be extended to shear-thinning or shear-thickening suspending fluids along the same lines.

\section{Acknowledgements}

This work was undertaken under the auspices of the ANR projects 'Rhéologie des suspensions concentrées' (ANR-08-BLAN-0048-CSD2) and 'Dense Particulate Systems' (ANR-13-IS09-0005-01), the 'Laboratoire d'Excellence Mécanique et Complexité' (ANR-11LABX-0092), the 'Initiative d'Excellence' A*MIDEX (ANR-11-IDEX-0001-02), and the COST Action MP1305 'Flowing Matter'. It also benefited from funding from 'Études et Productions Schlumberger'.

\section{References}

[1] A. Einstein, Annalen der Physik 17, 549 (1905)

[2] J. Stickel, R. Powell, Annual Review of Fluid Mechanics 37, 129 (2005)

[3] J. Morris, Rheologica Acta 48, 909 (2009)

[4] É. Guazzelli, J.F. Morris, A Physical Introduction to Suspension Dynamics (Cambridge University Press, 2012)

[5] M.M. Denn, J.F. Morris, Annual Review of Chemical and Biomolecular Engineering 5, 203 (2014)

[6] J. Morris, F. Boulay, Journal of Rheology 43, 1213 (1999) 
[7] F. Boyer, O. Pouliquen, É. Guazzelli, Journal of Fluid Mechanics 686, 5 (2011)

[8] E. Couturier, F. Boyer, O. Pouliquen, É. Guazzelli, Journal of Fluid Mechanics 686, 26 (2011)

[9] B. Snook, L.M. Davidson, J.E. Butler, O. Pouliquen, É. Guazzelli, Journal of Fluid Mechanics 758, 486 (2014)

[10] I. Zarraga, D. Hill, D. Leighton Jr, Journal of Rheology 44, 185 (2000)

[11] I. Zarraga, D. Hill, D. Leighton Jr, Journal of Rheology 45, 1065 (2001)

[12] A. Singh, P. Nott, Journal of Fluid Mechanics 490, 293 (2003)

[13] S.C. Dai, E. Bertevas, F. Qi, R.I. Tanner, Journal of Rheology 57, 493 (2013)

[14] T. Dbouk, L. Lobry, E. Lemaire, Journal of Fluid Mechanics 715, 239 (2013)

[15] C. Gamonpilas, J.F. Morris, M.M. Denn, Journal of Rheology 60, 289 (2016)

[16] S. Gallier, E. Lemaire, L. Lobry, F. Peters, Journal of Fluid Mechanics 799, 100 (2016)

[17] Y. Yurkovetsky, J. Morris, Journal of Rheology 52, 141 (2008)

[18] A. Deboeuf, G. Gauthier, J. Martin, Y. Yurkovetsky, J. Morris, Physical Review Letters 102, 108301 (2009)

[19] D. Prasad, H. Kytömaa, International Journal of Multiphase Flow 21, 775 (1995)
[20] F. Boyer, É. Guazzelli, O. Pouliquen, Physical Review Letters 107, 188301 (2011)

[21] S. Garland, G. Gauthier, J. Martin, J.F. Morris, Journal of Rheology 57, 71 (2013)

[22] D. Leighton, A. Acrivos, Journal of Fluid Mechanics 181, 415 (1987)

[23] A. Karnis, H. Goldsmith, S. Mason, Journal of Colloid and Interface Science 22, 531 (1966)

[24] B. Snook, J.E. Butler, É. Guazzelli, Journal of Fluid Mechanics 786, 128 (2016)

[25] P. Nott, J. Brady, Journal of Fluid Mechanics 275, 157 (1994)

[26] D. Lhuillier, Physics of Fluids 21, 023302 (2009)

[27] P.R. Nott, É. Guazzelli, O. Pouliquen, Physics of Fluids 23, 043304 (2011)

[28] K. Yeo, M.R. Maxey, Journal of Fluid Mechanics 682, 491 (2011)

[29] S. Dagois-Bohy, S. Hormozi, É. Guazzelli, O. Pouliquen, Journal of Fluid Mechanics 776, R2 (2015)

[30] B. Andreotti, Y. Forterre, O. Pouliquen, Granular Media: Between Fluid and Solid (Cambridge University Press, 2013)

[31] X. Chateau, G. Ovarlez, K.L. Trung, Journal of Rheology 52, 489 (2008)

[32] E. Lerner, G. Düring, M. Wyart, Proceedings of the National Academy of Sciences of the United States of America 109, 4798 (2012) 